\title{
PROPOSTA DE UTILIZAÇÃO DE LABORATÓRIOS VIRTUAIS PARA O ENSINO DE REDES DE COMPUTADORES: ARTICULANDO FERRAMENTAS, CONTEÚDOS E POSSIBILIDADES. (Fase I)
}

\author{
Gleizer Bierhalz Voss, PPGI, UFSM, gleizer.voss@gmail.com \\ Roseclea Duarte Medina, PPGI, UFSM, roseclea.medina@gmail.com \\ Érico M. H. Amaral, PPGIE/UFRGS, UNIPAMPA, ericohoffamaral@gmail.com \\ Fabrício Viero do Araújo, UFSM/ULBRA, rufos@terra.com.br \\ Felipe Becker Nunes, PPGI, UFSM, nunesfb@gmail.com \\ Taciano Balardin de Oliveira, PPGI, UFSM, tacianobalardin@gmail.com
}

\begin{abstract}
Resumo. O crescimento das TICs criou novas possibilidades na área da educação, exigindo o desenvolvimento de novas práticas pedagógicas que contribuam para o processo de ensino e aprendizagem. Esta fase do trabalho (Fase I) apresenta sugestões para a utilização de laboratórios virtuais (simuladores e demais ferramentas interativas) na disciplina de Redes de Computadores, de forma articulada, ou seja, considera os conteúdos a serem tratados, associados à ferramentas mais adequadas para a exploração de determinado assunto, assim como possibilidades de utilização. A pesquisa foi desenvolvida a partir de um estudo sobre os temas abordados nessa disciplina, bem como a análise e escolha das ferramentas que podem ser utilizadas nos conteúdos envolvidos.
\end{abstract}

Palavras-chave: ensino de redes de computadores, ferramentas interativas, simuladores, laboratórios virtuais.

\section{PROPOSAL USE OF VIRTUAL LABORATORIES FOR THE TEACHING OF COMPUTER NETWORKS: ARTICULATING TOOLS, CONTENT AND POSSIBILITIES. (Phase I)}

\begin{abstract}
The growth of ICT has created new possibilities in the area of education, requiring the development of new pedagogical practices that contribute to the process of teaching and learning. This phase of work (Phase I) presents suggestions for the use of virtual labs (simulators and other interactive tools) in the discipline of Computer Networks, pivotally, ie considers the content to be treated, associated with most appropriate tools for exploration of a subject, as well as potential uses. The research was developed from a study of the topics covered in this discipline, as well as analysis and selection of tools that can be used in content involved.
\end{abstract}

Keywords: teaching computer networking, interactive tools, simulators, virtual labs.

\section{INTRODUÇÃO}

O crescente desenvolvimento das tecnologias de comunicação e informação tem provocado mudanças nos mais diversos setores da sociedade (e.g, indústria, comércio, agricultura, entre outros). $\mathrm{Na}$ área da educação, essas tecnologias vêm quebrando o paradigma tradicional do processo de ensino e aprendizagem, trazendo a necessidade de novas práticas pedagógicas e exigindo dos professores outra maneira de ensinar, utilizando os novos recursos oferecidos pelo avanço da tecnologia da informação. Vale ressaltar a importância da concepção e desenvolvimento de softwares educacionais como instrumentos potencializadores da construção do conhecimento, visando uma 
transformação de paradigmas educacionais e possibilitando práticas pedagógicas inovadoras (Falkembach, 2005).

O ensino de temas técnicos na área de redes de computadores envolve conceitos difíceis de serem entendidos na forma pedagógica tradicional, ou seja, de forma estática. Entretanto, utilizando tecnologias digitais é possível facilitar o aprendizado do aluno com atividades, simulações e exercícios que complementam a fixação dos assuntos abordados.

O projeto "Lab-RC" está dividido em duas fases. Na primeira, descrita neste artigo, foi realizada a pesquisa das ferramentas, os testes das suas funcionalidades e apresentadas sugestões para a utilização dessas ferramentas de forma didática na disciplina, com o objetivo de estimular o aluno a refletir sobre os temas desenvolvidos a fim de atingir uma aprendizagem significativa. A segunda fase será a validação dessas ferramentas com alunos em sala de aula, considerando três instituições e cursos de ensino superior envolvidos neste projeto.

O desenvolvimento deste trabalho se justifica, entre outros motivos, pela necessidade de um novo cenário educacional no qual é fundamental conhecer novas formas de aprender e de ensinar, de comunicar e de representar conhecimento para atender aos alunos que pertencem a esta geração. Conforme Dutra (2002), devem ser utilizadas abordagens centradas no aluno, tendo por objetivo a preparação de profissionais que possuam, além do conhecimento técnico em redes, a habilidade de aplicar estes conhecimentos em problemas reais e a buscar o aperfeiçoamento por meio da aprendizagem e do trabalho colaborativo. Assim, este artigo tem como objetivo apresentar sugestões para o desenvolvimento da disciplina de Redes de Computadores, contemplando a utilização, de forma articulada, de laboratórios virtuais (softwares, ferramentas computacionais e simuladores) considerando conteúdos a serem tratados, associando-os às ferramentas mais adequadas para a exploração e reflexão de determinado assunto, bem como possibilidades de utilização.

\section{REVISÃO BIBLIOGRÁFICA}

\subsection{Redes de Computadores}

Redes de computadores podem ser definidas como "Um conjunto de computadores autônomos interconectados por uma única tecnologia." (Tanenbaum, 2003, p. 2). Para Torres (2001, p. 7), a implementação de uma rede de computadores tem como objetivo aumentar a produtividade do trabalho através da troca de dados (arquivos, e-mails, etc.) e do compartilhamento de periféricos (impressoras, modems, unidades de CD-ROM, etc.).

O avanço tecnológico vivido nas últimas décadas tanto nas áreas das Telecomunicações quanto na área da Tecnologia da Informação impulsionou o progresso na utilização das redes de computadores. Além disso, a globalização observada na economia, na política e no comportamento das nações trouxe necessidades em que o uso das redes tornou-se indispensável, fazendo com que milhões de pessoas passassem a utilizá-la para fins comerciais e domésticos. O crescimento exponencial das redes de computadores e da sua utilização, considerando também a complexidade e diversidade de tecnologias envolvidas, exige uma sólida formação profissional, bem como uma atualização constante.

\subsection{Ensino de Redes de Computadores}


O ensino de temas técnicos envolve os conteúdos a serem ensinados, os procedimentos pedagógicos que serão utilizados, a estratégia de como fazê-lo e a interação entre o professor e o aluno. Para Pinheiro et al. (2009), a utilização de simuladores pode representar situações e comportamentos difíceis de serem representados na vida real, servindo no preparo e treinamento de alunos para que aprendam a lidar com situações reais. Ele afirma ainda que é possível trabalhar com equipamentos que custam centenas de milhares de dólares, em ambientes fidedignos ao real, porém sem o risco de danificálos, ou seja, esses simuladores além de contribuir para a simplificação da instrução e do treinamento podem ainda proporcionar uma economia considerável referente ao custo com aquisição e manutenção de equipamentos.

Medina (2006), ao discutir o uso de materiais de ensino de redes de computadores visando a uma aprendizagem significativa, escreve que o uso de ferramentas como meio de ensino implica mais do que contato direto e observação de objetos e eventos:

\begin{abstract}
Envolve, também, a experiência de descoberta e a relação com aspectos do processo científico, tais como formação e testagem de hipóteses, projeto e realização de experimentos, controle e manipulação de variáveis e possibilidades de fazer inferências a partir de dados. Destaca que a experiência das ferramentas é útil e necessária para a compreensão da ciência e enfatiza que estas devem ser cuidadosamente integradas com o conteúdo abordado na disciplina, ou seja, devem lidar com metodologia ligada ao assunto do curso e não com experiências escolhidas somente por serem adequadas para ilustrar várias estratégias de descoberta. (Ausubel, 1980 citado por Medina, 2006, p. 86)
\end{abstract}

Ressalta-se que um dos principais problemas identificados nessas atividades é a falta de utilização dessas ferramentas de forma pontual, na qual são realizadas atividades predominantemente teóricas, que não retratam a realidade prática do ensino de redes de computadores. Com base nisso, diversos trabalhos têm sido desenvolvidos com o propósito de buscar atender a estas necessidades, por exemplo, em (Dutra, 2002), (Medina, 2004), (Pinheiro et al., 2009) e (Rauen, 2003) são apresentadas alternativas para melhorar o ensino e o aprendizado com a utilização de simuladores e laboratórios virtuais.

O estudo de ferramentas e conteúdos para a aplicação de laboratórios virtuais no ensino da disciplina de Redes de Computadores é uma alternativa que visa aprimorar e facilitar o processo de aprendizagem dos alunos em sala de aula. Através da utilização de ferramentas que atribuam um maior grau de praticidade às atividades programáticas do conteúdo da disciplina de redes, pretende-se aperfeiçoar o conhecimento obtido pelos alunos em sala de aula.

\title{
2.3 Ferramentas
}

A seguir são listadas as ferramentas utilizadas, bem como uma breve descrição sobre cada uma delas, exibindo os seus recursos principais. Esta listagem foi elaborada a partir da instalação e testes das ferramentas, e considerando as características básicas descritas em suas respectivas páginas $W e b$.

Cisco Packet Tracer (Cisco, 2011) - é uma ferramenta de aprendizado que suporta uma vasta gama de simulações físicas e lógicas, além de fornecer ferramentas de visualização para ajudar a entender os trabalhos internos de uma rede.

Cnet Network Simulator (Cnet, 2011) - este simulador permite a exploração e experimentação de uma série de protocolos das principais camadas de redes, em 
ambientes compostos pela combinação de redes com diferentes características, como redes de longa distância (WAN), redes locais (LAN) ou ainda redes locais sem fio (WLAN).

GNS3 - Graphical Network Simulator (Gns, 2011) - é um simulador gráfico de redes que permite a simulação de redes complexas. $O$ projeto é desenvolvido em código aberto, sendo compatível com todas as plataformas.

Jimsim (Jimsim, 2011) - é um aplicativo que emula roteadores conectados através de redes virtuais. Permite conectar-se aos roteadores através de um programa de telnet qualquer.

NCTUns - NCTU Network Simulator (Nctuns, 2011) - é um simulador de redes extensível e de alta fidelidade capaz de simular múltiplos protocolos utilizados tanto em redes IP fixas como wireless.

NetSimK (Netsimk, 2011) - esse simulador permite criar redes com roteadores e Swicthes Cisco, além de possuir a função de Calculadora IP integrada.

Network Simulator - ns-2 (Network Simulator, 2011) - é um simulador para eventos discretos orientado para a pesquisa em redes de computadores.

OPNET - Optimized Network Engineering Tools (Opnet, 2011) - fornece um ambiente virtual para modelagem, análise e previsão do desempenho das infraestruturas de TI, incluindo aplicações, servidores e tecnologias de rede.

SiReViW - Simulador Rede Virtual para Web 2.0 (Neto e da Silva, 2009) - é um programa que auxilia no aprendizado e na montagem de projetos de redes de computadores. Permite ao usuário montar uma rede LAN, MAN ou WAN, ou ainda unir essas redes através de roteadores.

\section{DESENVOLVIMENTO}

\subsection{Análise dos planos de ensino}

O desenvolvimento inicial deste trabalho foi baseado no estudo sobre os temas abordados na disciplina de Redes de Computadores em nível de graduação. Nesta etapa do trabalho (Fase 1) foram analisadas as ementas dessa disciplina de três (3) instituições de ensino.

Essas ementas foram estudadas para verificar quais são os principais conteúdos vistos durante o andamento da disciplina e seus respectivos objetivos. A avaliação foi realizada através da comparação, considerando os objetivos, carga horária e conteúdos apresentados. Inicialmente foram analisados os objetivos gerais, conforme mostrado no Quadro 1.

\section{Quadro 1 - Comparativo dos objetivos da disciplina}

\begin{tabular}{|c|l|}
\hline Instituição & \multicolumn{1}{c|}{ Objetivos gerais } \\
\hline UFSM & $\begin{array}{l}\text { Reconhecer e aplicar as formas de distribuição da informação e dos protocolos de } \\
\text { acesso a redes de computadores. }\end{array}$ \\
\hline ULBRA & $\begin{array}{l}\text { Fornecer ao aluno conceitos de comunicação de dados enfatizando as camadas } \\
\text { físicas e de enlace da arquitetura hierárquica de redes. }\end{array}$ \\
\hline UNIFRA & $\begin{array}{l}\text { Conhecer, de forma abrangente, as diversas tecnologias de rede com vistas a } \\
\text { avaliar, projetar, implementar e gerir estes ambientes de forma otimizada. }\end{array}$ \\
\hline
\end{tabular}

Após a análise dos objetivos da disciplina, foi realizada a comparação dos principais conteúdos abordados em cada uma das instituições. Esse comparativo pode ser visualizado no Quadro 2. Em relação aos conteúdos das ementas, é possível visualizar que a maioria dos temas são abordados nas 3 (três) instituições de ensino. 
Quadro 2 - Comparativo dos conteúdos por instituição de ensino

\begin{tabular}{|l|c|c|c|}
\hline \multicolumn{1}{|c|}{ Conteúdos } & UFSM & ULBRA & UNIFRA \\
\hline Introdução (Histórico; Conceitos) & $\mathrm{X}$ & $\mathrm{X}$ & $\mathrm{X}$ \\
\hline Estrutura e topologias de redes & $\mathrm{X}$ & $\mathrm{X}$ & $\mathrm{X}$ \\
\hline Camada de rede & $\mathrm{X}$ & $\mathrm{X}$ & \\
\hline Camada de transporte & $\mathrm{X}$ & $\mathrm{X}$ & \\
\hline Camada de aplicação & $\mathrm{X}$ & $\mathrm{X}$ & $\mathrm{X}$ \\
\hline Gerenciamento de Redes & $\mathrm{X}$ & $\mathrm{X}$ & \\
\hline Segurança de redes & $\mathrm{X}$ & & \\
\hline Projeto de redes & $\mathrm{X}$ & $\mathrm{X}$ & $\mathrm{X}$ \\
\hline Redes de alta velocidade & & $\mathrm{X}$ & $\mathrm{X}$ \\
\hline $\begin{array}{l}\text { Serviços de rede, Redes sem fio, Virtualização e } \\
\text { Governança }\end{array}$ & & $\mathrm{X}$ & \\
\hline
\end{tabular}

Considerando a descrição dos objetivos, associados aos conteúdos em comum apresentados nas ementas, foram escolhidos quais temas deveriam ser cobertos pelas ferramentas, sendo eles: Estrutura e topologias de redes, Modelo TCP/IP e Projeto de redes. O conteúdo referente à Introdução (Histórico, Conceitos), apesar de ser comum aos três planos de ensino, não foi abordado de maneira específica pelas ferramentas, pois se trata de um tema com base estritamente teórica. Contudo, as teorias estudadas poderão ser identificadas no decorrer das demais atividades propostas.

$\mathrm{O}$ tema modelo TCP/IP ou pilha TCP/IP, embora não tenha sido explicitamente descrito nos planos de ensino, está presente nos 3 (três) através das suas camadas de rede, transporte e aplicação.

\section{RESULTADOS}

As ferramentas foram estudadas com o objetivo de conhecer suas funcionalidades, potencialidades e limitações, bem como auxiliar na definição de possíveis estratégias de utilização das mesmas, como apoio no ensino da disciplina de Redes de Computadores. O Quadro 3 apresenta resumidamente o resultado da análise realizada para estabelecer uma correspondência entre os conteúdos abordados na disciplina e quais ferramentas podem ser utilizadas para auxiliar na compreensão e reflexão sobre determinado assunto.

Quadro 3 - Relação entre temas propostos e ferramentas

\begin{tabular}{|c|c|c|c|c|c|}
\hline Temas propostos & Packet Tracer & Jim Sim & Net Simk & OPNET IT Guru & SireViW \\
\hline Topologias de redes & $\mathrm{X}$ & & $\mathrm{X}$ & $\mathrm{X}$ & $\mathrm{X}$ \\
\hline Modelo TCP/IP & $\mathrm{X}$ & $\mathrm{X}$ & $\mathrm{X}$ & $\mathrm{X}$ & $\mathrm{X}$ \\
\hline Projeto e gerência de redes & $\mathrm{X}$ & & $\mathrm{X}$ & $\mathrm{X}$ & $\mathrm{X}$ \\
\hline
\end{tabular}

No Quadro 3 visualiza-se que a maioria das ferramentas atende, de uma forma ou de outra, todos os temas selecionados. Isso gera dúvidas e até dificuldades para os professores da disciplina ao determinarem qual seria a mais apropriada ou adequada para um tema. Sendo assim, ensejando contribuir neste processo, as próximas seções apresentam propostas de utilização das ferramentas de forma didático-articulada na disciplina de redes.

\subsection{Tema "Topologias de Redes"}


Para esse tema, podem ser utilizadas as ferramentas: Cisco Packet Tracer, Net Simk, OPNET IT GURU ou SireViW, propondo aos alunos que criem as topologias acima citadas, já que todas elas dispõem desta funcionalidade.

Por exemplo, ao utilizar o Cisco Packet Tracer, espera-se auxiliar os alunos a desenvolverem suas habilidades práticas em topologias de rede. Além disso, esta ferramenta suporta uma vasta gama de simulações físicas e lógicas, que permite visualizações e avaliação de recursos que facilitam a aprendizagem de conceitos complexos a respeito da tecnologia de redes de computadores (e.g, roteamento estático e dinâmico, funcionamento dos protocolos, etc.), e ainda desenvolver habilidades, tais como: tomada de decisão, pensamento crítico e resolução de problemas. Além disso, essa ferramenta permite visualizações que ajudam a entender os trabalhos internos de uma rede (e.g, subnetting, encaminhamento de pacotes, entre outros). Suas atividades consistem em simulações de rede, jogos, atividades e desafios, permitindo ensinar e demonstrar conceitos técnicos e projetos de sistemas de rede. No entanto, esta ferramenta está disponível gratuitamente apenas para instrutores, alunos, ex-alunos e administradores registrados na Networking Academy.

Já o SiReViW (Simulador Rede Virtual para Web 2.0), permite que o aluno tenha uma visão de como funcionam as redes, a interligação e a interação entre elas, beneficiando o aprendizado. Esta ferramenta permite ao usuário montar uma rede LAN, MAN ou WAN, ou ainda unir essas redes através de roteadores. Contudo, necessita de outros softwares previamente instalados, como o servidor de aplicações Java para Web "Tomcat" e o navegador "FireFoxPortable". Além disso, não possui tantas funcionalidades quanto às demais ferramentas escolhidas.

As Figuras 1 e 2 ilustram topologias montadas a partir da utilização destas ferramentas, nessas topologias é possível visualizar alguns equipamentos, como: PCs (Personal Computers), switches, roteadores, entre outros.

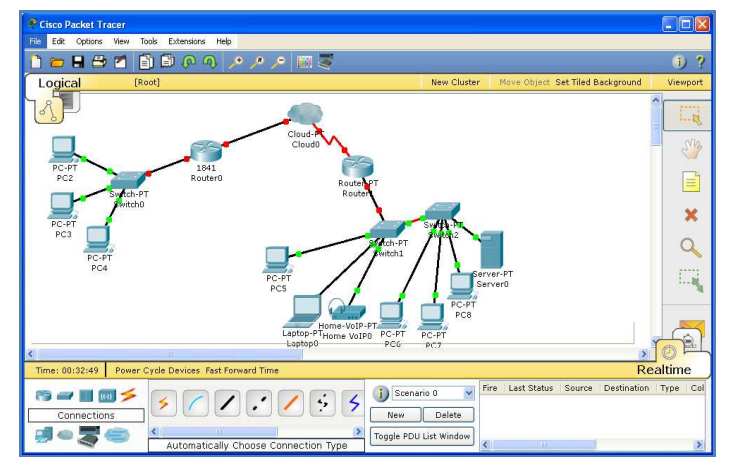

Figura 1 - Exemplo de simulação com o Cisco Packet Tracer

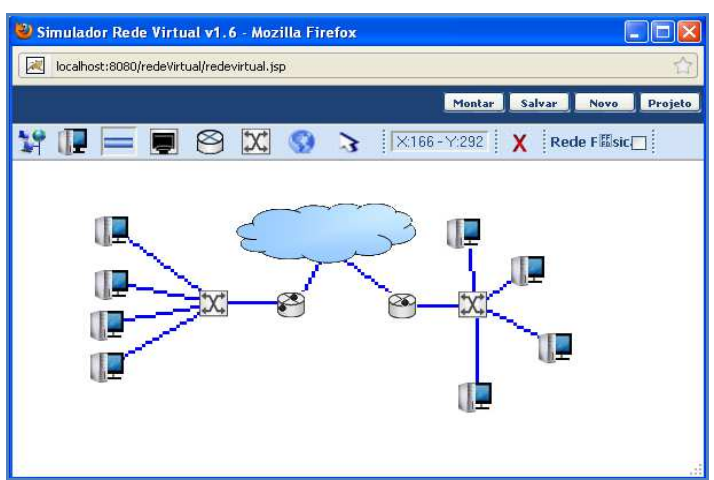

Figura 2 -Topologia montada utilizando a ferramentas SiReViW

Com o desenvolvimento dessa atividade, espera-se que o aluno esteja apto a identificar e compreender: os principais tipos de topologias; como se dá a conexão entre os dispositivos de rede; avaliar a rede quanto à modularidade, confiabilidade e desempenho.

\subsection{Tema "Modelo TCP/IP"}

Com relação a esse assunto, é possível propor uma série de atividades baseadas nas ferramentas elencadas. Uma delas é simular o acesso remoto aos roteadores da ferramenta JimSim através da utilização do protocolo de terminal virtual telnet. Outra 
sugestão de atividade é a proposta da montagem de uma ou mais redes, onde sejam configurados os endereços IPs de cada máquina, utilizando, por exemplo, a ferramenta NetSimk.

O Jimsim emula três roteadores com uma interface semelhante às do IOS (Internetwork Operating System) CISCO. Esses roteadores apresentam apenas interfaces Ethernet, que são conectadas a quatro redes virtuais separadas. Para realizar as simulações, é possível utilizar um programa de telnet qualquer para acessar e configurar os roteadores, permitindo que iniciantes aprendam a utilizar esses equipamentos. Vale ressaltar que esta ferramenta é livre apenas para uso pessoal, para ser utilizada em classe é necessário a autorização do autor. Além disso, a versão testada é bastante limitada na quantidade de recursos, o suporte ao EIGRP é muito básico, e a tabela de roteamento eventualmente apresenta informações não fidedignas, exigindo assim atenção e eventual correção da informação pelo professor.

Já com o NetSimk, é possível criar redes com roteadores e switches Cisco, porém, sua principal característica é a capacidade de gerar erros aleatórios nas configurações criadas para auxiliar no estudo da resolução de problemas (troubleshooting). Ao mesmo tempo, permite ao usuário criar as próprias topologias a partir de um cenário em branco, ou ainda utilizar topologias padrão disponíveis no menu principal. Possui também uma calculadora IP integrada, facilitando a configuração dos endereços IPs e a respectiva compreensão e aprendizado de um assunto considerado bastante complexo para os alunos. No entanto, está disponível gratuitamente apenas na versão de avaliação (com prazo definido), e não permite configurar os protocolos EIGRP e OSPF.

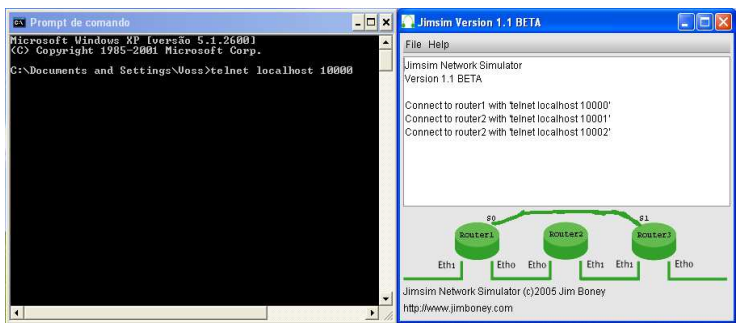

Figura 3 - Acesso telnet e tela inicial do JimSim

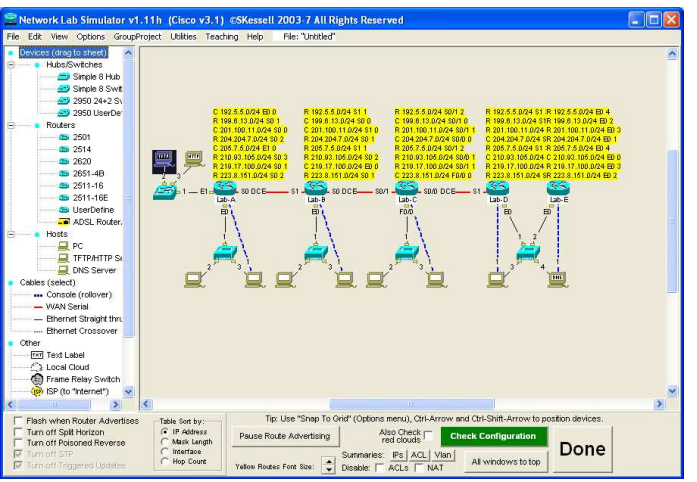

Figura 4 - Net Simk exibindo Layout padrão Cisco CCNA

A Figura 3 mostra a tela inicial do Jim Sim e um dos roteadores sendo acessado via telnet no Windows. Já a Figura 4 apresenta uma topologia padrão Cisco CCNA com as respectivas tabelas de roteamento de cada um dos roteadores destacadas em amarelo.

\subsection{Tema "Projeto e Gerência de Redes"}

Normalmente, após a apresentação de todos os temas/conteúdos, é solicitada aos alunos a construção de um Projeto de Redes, abordando todos os temas da disciplina como: Comunicação de dados, Topologias, Cabeamento estruturado, Padrões de rede, Protocolo TCP/IP, Servidores, Redes sem fio, Virtualização e Gerência de redes.

Para viabilizar a elaboração desse projeto, podem ser utilizadas as ferramentas: Cisco Packet Tracer, Net Simk, OPNET IT Guru ou SireViW. Através dessas 
ferramentas é possível desenvolver essa atividade de uma maneira ampla e objetiva, aproximando-se das necessidades que seriam encontradas em um projeto real.

Ao utilizar a ferramenta OPNET IT Guru Academic Edition, por exemplo, é possível realizar simulações de redes com configurações fiéis à realidade, possibilitando que sejam coletadas e analisadas diversas estatísticas a respeito da simulação, considerando que esta ferramenta foi desenvolvida para ser utilizada em sala de aula como forma de complementar os exercícios de laboratório específicos para cursos de redes, juntamente com manuais de laboratório e livros texto.

A Figura 5 ilustra a criação de um cenário utilizando esta ferramenta, à esquerda da figura encontra-se o cenário principal com alguns objetos inseridos, à direita da figura, conforme indicado pela seta, está a paleta de objetos. Esta paleta contém uma grande variedade de objetos que podem ser inseridos no cenário principal, entre eles: roteadores, switches, firewalls, links de comunicação, etc.

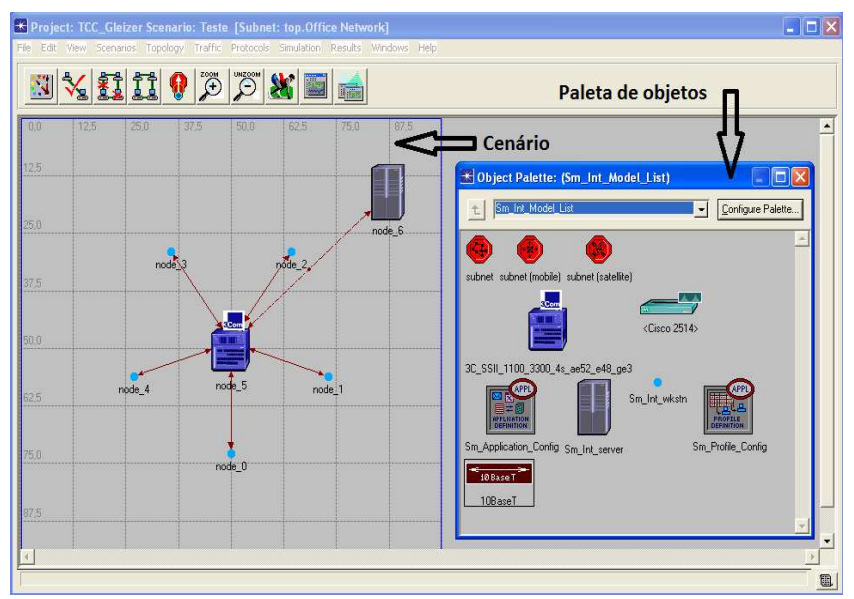

Figura 5 - Criação de um cenário no OPNET

A criação desse projeto pode ser desenvolvido desde o início da disciplina, conforme o andamento dos conteúdos (e.g, comunicação de dados, topologias, etc.), bem como pode ser proposto como projeto final, abordando os temas de uma forma mais ampla.

\subsection{Ferramentas não selecionadas}

A partir do estudo sobre as ferramentas, da instalação e dos testes das mesmas, optou-se por não utilizar algumas daquelas listadas inicialmente na seção 2.3, ficando como alternativas para trabalhos futuros. Essas ferramentas também apresentam suas vantagens, no entanto algumas características apresentadas foram decisivas para a não utilização das mesmas nesta fase do trabalho, conforme listadas a seguir: Duas delas não possuem interface gráfica de usuário (GUI - Graphical User Interface) (Cnet e ns-2) e as outras duas (NCTUns e GNS-3) possuem restrições na sua utilização.

O simulador Cnet não roda em ambiente Windows, e mostra-se uma ferramenta complexa de instalar e realizar simulações, pois exige que o usuário crie scripts escritos no padrão ANSI da linguagem C, para posteriormente serem compilados. Ainda nesse sentido, para realizar uma simulação no $n s-2$, é necessário realizar a programação em duas linguagens: uma para a estrutura básica (protocolos, agentes, etc.) em $\mathrm{C}++\mathrm{e}$ outra para o uso como frontend através da OTCL (Object-oriented Tool Command Language). O processo de instalação dessas ferramentas, dependendo da versão do Linux utilizada pode ser uma tarefa árdua, principalmente para aqueles que não têm 
muita experiência nesse sistema operacional, pois é necessária a edição de vários arquivos de configuração. Além disso, a visualização de uma simulação requer a ferramenta NAM (Network Animator), e a análise dos resultados da simulação, demanda uma ferramenta que analise o arquivo Trace gerado pelo $n s$.

Já no GNS-3 é necessário ter o IOS do roteador disponível, caso contrário não é possível realizar simulações. Ressaltando que para baixar uma imagem do sistema operacional, é necessário possuir uma conta CCO (Cisco Connection Online) ativa. Por sua vez, o software NCTUns passou a ser comercializado devido ao seu grande sucesso, passando a ser chamado em sua versão comercial de EstiNet 7.0.

\section{CONSIDERAÇÕES FINAIS}

De acordo com o presente estudo é possível concluir que as ferramentas estudadas atendem os objetivos da disciplina de Redes de Computadores em nível de graduação. O resultado da análise e comparação entre os temas apresentados na disciplina e as ferramentas que possibilitam abordar tais temas, demonstraram que é viável a utilização dessas ferramentas de forma articulada, contribuindo assim para uma melhor reflexão e compressão dos temas envolvidos. Além disso, podem ser associadas a outras atividades considerando os demais conteúdos abordados na disciplina que não foram listadas neste trabalho, apoiando tanto atividades presenciais quanto à distância.

Através deste estudo chegou-se a conclusão de que as ferramentas Cisco Packet Tracer, Jim Sim, Net Simk, OPNET IT Guru e SireViW são as mais indicadas para servirem como suporte às atividades envolvendo os tópicos abordados nessa disciplina, considerando a praticidade de instalação, a facilidade de uso e aprendizado, além dos recursos oferecidos e as interfaces gráficas das mesmas.

A partir dos resultados obtidos nesta primeira fase, na próxima etapa desta proposta (Fase II), pretende-se avaliar em sala de aula, além da compreensão dos conceitos envolvidos com a utilização adequada das ferramentas/laboratórios virtuais, o grau de motivação dos alunos para o aprendizado, considerando as possibilidades do fazer e refazer, da diversidade de cenários e de testes possíveis, e quanto esta motivação impacta na sua formação cognitiva, ampliando as habilidades obtidas para a resolução de problemas reais.

Também na Fase II será elaborada uma lista de atividades mais específicas para uma ou mais das ferramentas escolhidas, a fim de validar as ferramentas a partir de testes com alunos, comparando, por exemplo, o desempenho entre os alunos que utilizaram a ferramenta " $\mathrm{X}$ " com os que utilizaram a ferramenta "Y", ou aqueles que não fizeram uso de nenhuma ferramenta.

Além disso, como trabalhos futuros, é possível realizar a adaptação dessa estratégia para o ensino da disciplina em cursos técnicos ou até mesmo de pósgraduação.

\section{Referências Bibliográficas}

Cisco. Course Catalog - Cisco Packet Tracer. 2011. Apresenta informações sobre o software, opções para download e guias para utilização. Disponível em: <http://www.cisco.com/web/learning/netacad/course_catalog/PacketTracer.html>.

Acesso em: 02 abr. 2011.

Cnet. Site da Universidade que desenvolve a ferramenta. 2011. Apresenta informações sobre o software, opções para download e guias para utilização.Disponível em: <http://www.csse.uwa.edu.au/cnet/>. Acesso em: 25 mai. 2011. 
DUTRA, Renato Luís de Souza. AAERO: Ambiente de Aprendizado para o Ensino de Redes de Computadores Orientado a Problemas. Disponível em: <http://hdl.handle.net/10183/1892>. Acesso em: 02 abr. 2011.

FALKEMBACH, Gilse A. M. Concepção e Desenvolvimento de Material Educativo Digital. Disponível em: <http://seer.ufrgs.br/renote/article/view/13742/7970>. Acesso em: 11 jun. 2011.

GNS3. Site da ferramenta. 2011. Apresenta informações sobre o software, opções para download e guias para utilização. Disponível em: <http://www.gns3.net/>. Acesso em: 22 abr. 2011.

Jimsim. About Jimsim. 2011. Apresenta informações sobre o software, opções para download e guias para utilização. Disponível em: <http://www.jimboney.com/about.php>. Acesso em: 22 abr. 2011.

MEDINA, Roseclea D. ASTERIX - Aprendizagem Significativa e Tecnologias aplicadas no Ensino de Redes de computadores: Integrando e eXplorando possibilidades. Tese de Doutorado, UFRGS, 2004. 174 p.

NCTUns. Site da ferramenta. 2011. Apresenta informações sobre o software, opções para download e guias para utilização. Disponível em: <http://nsl.csie.nctu.edu.tw/nctuns.html>. Acesso em: 02 abr. 2011.

NETO, Almerindo N. R.; DA SILVA, Gilmar N. Simulador Rede Virtual para Web 2.0. 2009. Disponível em: 〈http://goo.gl/hGAA4> Acesso em: 12 out. 2011.

NetSimK. What is NetSimK?. 2011. Apresenta informações sobre o software, opções para download e guias para utilização. Disponível em: <http://netsimk.com/>. Acesso em: 14 mai. 2011.

Network Simulator. Site da Universidade que desenvolve a ferramenta. 2011. Apresenta informações sobre o software, opções para download e guias para utilização.Disponível em: 〈http://www.isi.edu/nsnam/ns/>. Acesso em: 02 abr. 2011.

OPNET. Site da ferramenta. 2011. Apresenta informações sobre o software, opções para download e guias para utilização. Disponível em: <http://www.opnet.com/university_program/itguru_academic_edition/index.html>.

Acesso em: 14 mai. 2011.

PINHEIRO, Ricardo P.; LINS, Fernando A. A.; MELO, Jeane C. B. de. A Utilização de Simulação no Ensino de Redes de Computadores. Disponível em: <http://www.eventosufrpe.com.br/jepex2009/cd/resumos/R0311-1.pdf>. Acesso em: 18 jun. 2011.

RAUEN, Tânia Regina S. Uma Abordagem Alternativa para Ensino de Redes de Computadores. Dissertação de Mestrado, UFSC, 2003. 85 p.

TANENBAUM, Andrew S. Redes de computadores. Tradução Vandemberg D. de Souza. - Rio de Janeiro : Elsevier, 2003 - $6^{\text {a }}$ Reimpressão.

TORRES, Gabriel. Redes de Computadores Curso Completo. - Rio de Janeiro: Axcel Books, 2001. 